\title{
Lie groups as multiplication groups of topological loops
}

\author{
Ágota Figula
}

\begin{abstract}
In this short survey we give some new results about the question whether or not a Lie group can be represented as the multiplication group of a 3-dimensional topological loop. We deal with the classes of quasi-simple Lie groups and nilpotent Lie groups.
\end{abstract}

2010 Mathematics Subject Classification: 57S20, 57M60, 22A30, 22E25, 20N05

Key words and phrases: multiplication group of loops, topological transformation group, quasi-simple Lie groups, nilpotent Lie groups

\section{Introduction}

A loop $(L, \cdot)$ is a quasigroup with identity element $e \in L$. The left translations $\lambda_{a}: y \mapsto a \cdot y$ and the right translations $\rho_{a}: y \mapsto y \cdot a, a \in L$, are bijections of $L$. The group generated by all left and right translations of $L$ is called the multiplication group $\operatorname{Mult}(L)$ of $L$. The stabilizer of $e \in L$ in the group $\operatorname{Mult}(L)$ is the inner mapping group $\operatorname{Inn}(L)$ of $L$. The multiplication group and the inner mapping group of a loop $L$ are important tools for research in loop theory since there are strict relations between the structure of the groups $\operatorname{Mult}(L), \operatorname{Inn}(L)$ and that of $L$. If the group $\operatorname{Mult}(L)$ is simple, then the loop $L$ is also simple and the group $\operatorname{Inn}(L)$ is a maximal subgroup of $M u l t(L)$ (cf. [1], [4], [15]). The nilpotency of the group $M u l t(L)$ implies the centrally nilpotency of $L$ (cf. [4]).

The subgroup $G_{l}$ of $\operatorname{Mult}(L)$ generated by all left translations of $L$ is the group of left translations of $L$. Topological loops $L$ such that the groups $G_{l}$ of $L$ are Lie groups have been studied in [14] and they are treated as continuous sharply transitive sections $\sigma: G_{l} / H_{l} \rightarrow G_{l}$, where $H_{l}$ is the stabilizer of the identity element $e \in L$ in $G_{l}$.

For infinite groups in any studied category there is till now only one feasible criterion for the decision whether a group $\Gamma$ is the multiplication group of a loop $L$, namely the criterion of Niemenmaa and Kepka ([15]).

Proposition 1. A group $\Gamma$ is the multiplication group of $L$ if and only if there exists a maximal subgroup $K$ of $\Gamma$ containing no non-trivial proper normal subgroup of $\Gamma$ and two left transversals $A$ and $B$ of $K$ in $\Gamma$ such that $a^{-1} b^{-1} a b \in K$ for every $a \in A$ and $b \in B$ and the set $\{A, B\}$ generates $\Gamma$. 
This criterion has been successfully applied in particular in the case of Lie groups. The multiplication group $\operatorname{Mult}(L)$ of a topological loop $L$ is mostly a differentiable transformation group of infinite dimension. This is the case for every 1-dimensional topological loop ([14], Theorem 18.18, p. 248). For 2-dimensional topological loops $L$ the group $M u l t(L)$ is a Lie group precisely if it is an elementary filiform Lie group $\mathcal{F}_{n}$ with $n \geq 4$, i. e. if the Lie algebra $\operatorname{mult}(\mathbf{L})$ of $\operatorname{Mult}(L)$ has a basis $\left\{e_{1}, \cdots, e_{n}\right\}$ such that $\left[e_{1}, e_{i}\right]=e_{i+1}$ for $2 \leq i \leq n-1$ ([9], Theorem 1, p. 420).

In contrast to this there does not exist 3-dimensional connected simply connected topological loop $L$ having an elementary filiform Lie group as its multiplication group (cf. Proposition 5). The 4-dimensional indecomposable Lie groups are not multiplication groups of $L$. There are only two classes of the 5-dimensional nilpotent Lie groups which are multiplication groups of $L$. The corresponding loops $L$ are centrally nilpotent of class 2 (cf. Proposition 6).

In the last section we treat quasi-simple Lie groups acting transitively and effectively on 3-dimensional manifolds. None of the at most 8-dimensional quasi-simple Lie groups occurs as the multiplication group of a connected topological proper loop. However, the group $S L_{4}(\mathbb{R})$ is the multiplication group of connected topological loops homeomorphic to $S^{3}$.

\section{Preliminaries}

A binary system $(L, \cdot)$ is called a loop if there exists an element $e \in L$ such that $x=e \cdot x=x \cdot e$ holds for all $x \in L$ and the equations $a \cdot y=b$ and $x \cdot a=b$ have precisely one solution, which we denote by $y=a \backslash b$ and $x=b / a$. A loop $L$ is proper if it is not a group.

The kernel of a homomorphism $\alpha:(L, \cdot) \rightarrow\left(L^{\prime}, *\right)$ of a loop $L$ into a loop $L^{\prime}$ is a normal subloop $N$ of $L$. The centre $Z(L)$ of a loop $L$ consists of all elements $z$ which satisfy the equations $z x \cdot y=z \cdot x y, x \cdot y z=x y \cdot z, x z \cdot y=$ $x \cdot z y, z x=x z$ for all $x, y \in L$. If we put $Z_{0}=e, Z_{1}=Z(L)$ and $Z_{i} / Z_{i-1}=Z\left(L / Z_{i-1}\right)$, then we obtain a series of normal subloops of $L$. If $Z_{n-1}$ is a proper subloop of $L$ but $Z_{n}=L$, then $L$ is centrally nilpotent of class $n$. In [4] it was proved that if $\operatorname{Mult}(L)$ is a nilpotent group of class $n$, then $L$ is centrally nilpotent of class at most $n$.

The connections between the normal subgroup structure of Mult $(L)$ and the normal subloop structure of $L$ are the following. Let $L$ be a loop with multiplication group $\operatorname{Mult}(L)$ and identity element $e$. Let $N$ be a normal subloop of $L$ and $M(N)$ be the set $\{m \in \operatorname{Mult}(L) ; x N=m(x) N$ for all $x \in L\}$. Then $M(N)$ is a normal subgroup of $\operatorname{Mult}(L)$ containing the multiplication group $\operatorname{Mult}(N)$ of the loop $N$ and the multiplication group of the factor loop $L / N$ is isomorphic to $\operatorname{Mult}(L) / M(N)$. Conversely, for every normal subgroup $\mathcal{N}$ of $\operatorname{Mult}(L)$ the orbit $\mathcal{N}(e)$ is a normal subloop of $L$. Moreover, $\mathcal{N} \leq M(\mathcal{N}(e))$. (cf. [1], Theorems 3, 4 and 5 and in [5], IV.1, Lemma 1.3). 
Let $L$ be a loop and let $G_{l}$, respectively $G_{r}$ the group of left, respectively of right translations of $L$. We have $G_{l}=G_{r}=\operatorname{Mult}(L)$ if and only if for the stabilizers $H_{l}$, respectively $H_{r}$ of $e \in L$ in $G_{l}$, respectively in $G_{r}$ one has $H_{l}=H_{r}=\operatorname{Inn}(L)$ and for all $x \in L$ the map $f(x): y \mapsto \lambda_{x}^{-1} \lambda_{y} x: L \rightarrow L$ is an element of $\operatorname{Inn}(L)$.

A loop $L$ is called topological if $L$ is a topological space and the binary operations $(x, y) \mapsto x \cdot y,(x, y) \mapsto x \backslash y,(x, y) \mapsto y / x: L \times L \rightarrow L$ are continuous. Every connected topological loop $L$ having a Lie group $G_{l}$ as the group of left translations of $L$ is obtained on a homogeneous space $G_{l} / H_{l}$, where $H_{l}$ is a closed subgroup of $G_{l}$ with $C_{G_{l}}\left(H_{l}\right)=1$ and $\sigma: G_{l} / H_{l} \rightarrow G_{l}$ is a continuous section such that $\sigma\left(H_{l}\right)=1 \in G_{l}$, the subset $\sigma\left(G_{l} / H_{l}\right)$ generates $G_{l}$ and the set $\sigma\left(G_{l} / H_{l}\right)$ operates sharply transitively on $G_{l} / H_{l}$, which means that to any $x H_{l}$ and $y H_{l}$ there exists precisely one $z \in \sigma\left(G_{l} / H_{l}\right)$ with $z x H_{l}=y H_{l}$. The multiplication of $L$ on the manifold $G_{l} / H_{l}$ is defined by $x H_{l} * y H_{l}=\sigma\left(x H_{l}\right) y H_{l}$.

For any connected topological loop there exists universal covering which is simply connected (cf. [12], IX.1.).

A 2-dimensional connected simply connected loop $L_{\mathcal{F}}$ is called an elementary filiform loop if its multiplication group is an elementary filiform group $\mathcal{F}_{n}$, $n \geq 4([10])$.

A Lie group is called indecomposable if it is not the direct product of two proper ideals of positive dimension.

A quasi-simple connected Lie group is a connected Lie group $G$ such that any normal subgroup of $G$ is discrete and central in $G$. A connected loop $L$ is quasi-simple if any normal subloop of $L$ is discrete in $L$. According to [12], p. 216, all discrete normal subloops of a connected loop are central.

\section{Nilpotent Lie groups as multiplication groups of topological loops}

Lemma 3.3 in [9], p. 390, says the following.

Lemma 2. Let L be a 3-dimensional proper connected topological loop such that its multiplication group is a solvable Lie group. If $L$ is simply connected, then it is homeomorphic to $\mathbb{R}^{3}$.

Assume that the group $M u l t(L)$ of a 3-dimensional proper connected topological loop $L$ is nilpotent. For the centre $Z$ of $\operatorname{Mult}(L)$ one has $\operatorname{dim} Z \in$ $\{1,2\}$ (cf. Lemma 3.5. in [9], p. 391). By Theorem 11 in [1] the orbit $Z(e)$ is the centre $Z(L)$ of $L$. From Proposition 3.7. in [9], p. 392, one gets:

Proposition 3. Let $L$ be a 3-dimensional proper connected simply connected topological loop such that its multiplication group $M u l t(L)$ is a nilpotent Lie group and the centre $Z$ of Mult $(L)$ has dimension 2. Then Mult $(L)$ is a semidirect product of a group $Q \cong \mathbb{R}$ by the abelian group $M=Z \times \operatorname{Inn}(L) \cong$ $\mathbb{R}^{m}, m \geq 3$, where $\mathbb{R}^{2}=Z \cong Z(L)$. 
For every 1-dimensional connected subgroup $N$ of the centre $Z$ of $M u l t(L)$ the orbit $N(e)$ is a 1-dimensional connected normal subloop of $L$ containing in $Z(L)$ (cf. Lemma 3.6. (a) in [9], p. 392).

Proposition 4. Let L be a 3-dimensional proper connected simply connected topological loop such that its multiplication group $M u l t(L)$ is an indecomposable nilpotent Lie group. Then there exists a 1-dimensional central subgroup $N(e)$ of $L$ isomorphic to $\mathbb{R}$. Moreover, one of the following possibilities holds:

a) If the factor loop $L / N(e)$ is isomorphic to the abelian group $\mathbb{R}^{2}$, then the centre $Z$ of $M u l t(L)$ has dimension 1 and $N(e)=Z(e)=Z(L)$. Moreover, $\operatorname{Mult}(L)$ is a semidirect product of a group $Q \cong \mathbb{R}^{2}$ by the abelian group $P=Z \times \operatorname{Inn}(L) \cong \mathbb{R}^{m}, m \geq 2$.

b) If the factor loop $L / N(e)$ is isomorphic to a 2-dimensional elementary filiform loop $L_{\mathcal{F}}$, then there is a normal subgroup $S$ of Mult $(L)$ containing $N \cong \mathbb{R}$ such that the factor group $M u l t(L) / S$ is an elementary filiform Lie group $\mathcal{F}_{n}$ with $n \geq 4$.

Proposition 5. There does not exist any 3-dimensional connected simply connected topological loop having an elementary filiform Lie group as its multiplication group.

The classification of the indecomposable nilpotent Lie algebras of dimension 5 can be found in [11], pp. 167-168.

Proposition 6. Let $L$ be a connected simply connected topological proper loop of dimension 3 such that its multiplication group $M u l t(L)$ is a 5dimensional indecomposable nilpotent Lie group. Then $L$ contains a central subgroup $C \cong \mathbb{R}$ such that the factor loop $L / C \cong \mathbb{R}^{2}$. Moreover, the following Lie groups are the multiplication groups $M$ ult $(L)$ and the following subgroups are the inner mapping groups $\operatorname{Inn}(L)$ of $L$ :

1) $\operatorname{Mult}(L)_{1}$ is the direct product $\mathcal{F}_{3} \times{ }_{Z} \mathcal{F}_{3}$ with amalgamated centre $Z$ the multiplication of which is given by $g\left(q_{1}, z_{1}, w_{1}, x_{1}, y_{1}\right) g\left(q_{2}, z_{2}, w_{2}, x_{2}, y_{2}\right)=$

$$
g\left(q_{1}+q_{2}+z_{1} x_{2}+w_{1} y_{2}, z_{1}+z_{2}, w_{1}+w_{2}, x_{1}+x_{2}, y_{1}+y_{2}\right) .
$$

$\operatorname{Inn}(L)$ is the subgroup $\{g(0, z, w, 0,0), z, w \in \mathbb{R}\}$.

2) Mult $(L)_{2}$ is represented on $\mathbb{R}^{5}$ by the multiplication

$$
\begin{gathered}
g\left(x_{1}, y_{1}, q_{1}, z_{1}, w_{1}\right) g\left(x_{2}, y_{2}, q_{2}, z_{2}, w_{2}\right)= \\
g\left(x_{1}+x_{2}+q_{1} z_{2}+w_{1} y_{2}+\frac{w_{1}^{2} q_{2}}{2}, y_{1}+y_{2}+w_{1} q_{2}, q_{1}+q_{2}, z_{1}+z_{2}, w_{1}+w_{2}\right) .
\end{gathered}
$$

$\operatorname{Inn}(L)_{2}$ is one of the subgroups $\operatorname{Inn}(L)_{2,1}=\{g(0, y, q, 0,0), y, q \in \mathbb{R}\}$, $\operatorname{Inn}(L)_{2,2}=\{g(0, y, 0, z, 0), y, z \in \mathbb{R}\}$. 


\section{Quasi-simple Lie groups as multiplication groups of topological loops}

The following lemma is proved in [15], Lemma 2.6.

Lemma 7. Let $S$ be a proper subgroup of a simple group $K$ and let $A$ and $B$ be $S$-connected transversals in $K$. Then $S$ is maximal in $K$.

Proposition 8. If $L$ is a 3-dimensional connected simply connected topological loop having a quasi-simple Lie group as the multiplication group Mult $(L)$ of $L$, then one of the following cases can occur:

(a) $L$ is homeomorphic to $S^{3}$ and the group $M$ ult $(L)$ is one of the following Lie groups: $S L_{2}(\mathbb{C}), S U_{3}(\mathbb{C}, 1), S L_{4}(\mathbb{R}), S O_{5}(\mathbb{R}, 1), S p_{4}(\mathbb{R})$, the universal covering of $S L_{3}(\mathbb{R})$.

(b) $L$ is homeomorphic to $\mathbb{R}^{3}$ and the group $M$ ult $(L)$ is the group $P S L_{2}(\mathbb{C})$.

The proof of this Proposition can be found in [10], Proposition 3.2, p. 389.

Theorem 9. There does not exist connected topological loop $L$ such that its multiplication group $M u l t(L)$ is locally isomorphic to the group $P S L_{2}(\mathbb{C})$.

We consider the Lie groups which are locally isomorphic to $S L_{3}(\mathbb{R})$.

Lemma 10. If there exists a connected topological proper loop $L$ such that its multiplication group $M$ ult $(L)$ is locally isomorphic to $S L_{3}(\mathbb{R})$, then we have the following possibility: the group $M u l t(L)$ of $L$ as well as the group $G_{l}$ of $L$ is the group $S L_{3}(\mathbb{R})$, the stabilizer $\operatorname{Inn}(L)=H_{l}$ of $e \in L$ in the group $\operatorname{Mult}(L)=G_{l}$ is the subgroup $S O_{3}(\mathbb{R})$ and $L$ is homeomorphic to $\mathbb{R}^{5}$.

Theorem 11. There does not exist any connected topological loop L such that its multiplication group $M$ ult $(L)$ is locally isomorphic to the Lie group $S L_{3}(\mathbb{R})$.

Now we treat the Lie groups $G$ which are locally isomorphic to $P S U_{3}(\mathbb{C}, 1)$. From [3], Satz 1, p. 251 and [6], Section 5, p. 276, we obtain the following:

Proposition 12. Any connected closed maximal subgroup of $P S U_{3}(\mathbb{C}, 1)$ is one of the following groups

(1) $\mathrm{H}_{1}$ is isomorpic to the group $\operatorname{Spin}_{3} \times \mathrm{SO}_{2}(\mathbb{R})$,

(2) $\mathrm{H}_{2}$ is isomorphic to the 5-dimensional solvable group $N G$, where

$N=\left\{\left(\begin{array}{ccc}1 & z i & z \\ \bar{z} i & 1+i t-\frac{z \bar{z}}{2} & t+\frac{z \bar{z}}{2} \\ \bar{z} & t+\frac{z \bar{z}}{2} & 1-i t+\frac{z \bar{z}}{2}\end{array}\right) ; z \in \mathbb{C}, t \in \mathbb{R}\right\}$ and

$G=\left\{\left(\begin{array}{ccc}e^{-i k} & 0 & 0 \\ 0 & \frac{1}{2}\left(e^{-u}+e^{u}\right) e^{\frac{1}{2} i k} & \frac{1}{2}\left(e^{u}-e^{-u}\right) i e^{\frac{1}{2} i k} \\ 0 & \frac{1}{2}\left(e^{-u}-e^{u}\right) i e^{\frac{1}{2} i k} & \frac{1}{2}\left(e^{-u}+e^{u}\right) e^{\frac{1}{2} i k}\end{array}\right) ; k, u \in \mathbb{R}\right\}$,

(3) $\mathrm{H}_{3}$ is isomorphic to the group $S U_{2}(\mathbb{C}, 1) \times S O_{2}(\mathbb{R}) \cong S L_{2}(\mathbb{R}) \times S O_{2}(\mathbb{R})$,

(4) $\mathrm{H}_{4}$ is isomorphic to the group $S O_{0}(2,1) \cong P S L_{2}(\mathbb{R})$. 
Proposition 13. If there exists a connected topological proper loop $L$ such that its multiplication group $M u l t(L)$ is locally isomorphic to $P S U_{3}(\mathbb{C}, 1)$, then the following possibilities can occur:

(a) $L$ is homeomorphic to $\mathbb{R}^{4}$, the group $M u l t(L)$ is the group $P S U_{3}(\mathbb{C}, 1)$, the subgroup $\operatorname{Inn}(L)=H_{l}$ of Mult $(L)$ is the subgroup $H_{1}$ given in Proposition 12 (1).

(b) $L$ is homeomorphic to $S^{3}$, the group $M u l t(L)$ as well as the group $G_{l}$ of $L$ is the group PSU $(\mathbb{C}, 1)$, the subgroup $\operatorname{Inn}(L)=H_{l}$ of Mult $(L)$ is the subgroup $\mathrm{H}_{2}$ given in Proposition 12 (2).

Theorem 14. There does not exist a connected topological loop $L$ such that the group $M u l t(L)$ is the Lie group $\operatorname{PSU}_{3}(\mathbb{C}, 1)$.

Remark 15. Till now the only known quasi-simple Lie group which is the multiplication group of a 3-dimensional connected topological loop $L$ is the group $S L_{4}(\mathbb{R})$. Such loops $L$ are the multiplicative loops of locally compact connected topological quasifields $Q$ such that the kernel of $Q$ is the field of complex numbers and $Q$ has dimension 2 over its kernel. These quasifields $Q$ coordinatize non-desarguesian 8-dimensional topological translation planes and are determined by N. Knarr ([13], Section 6). Using the results of [13] we have proved that the multiplicative loops $Q^{*}$ are the direct products of $\mathbb{R}$ and a compact loop $\mathcal{S}$ homeomorphic to $S^{3}$ and having the group $S L_{4}(\mathbb{R})$ as its multiplication group (cf. [7]). There are two classes of such compact loops $\mathcal{S}$. One is related to Rees algebras (cf. [14], Section 29.2).

The simple Lie groups $P S L_{2}(\mathbb{C}), P S U_{3}(\mathbb{C}, 1), S L_{3}(\mathbb{R})$ are the groups $G_{l}$ topologically generated by all left translations for differentiable loops $L$. If the stabilizer $H_{l}$ of $e \in L$ in $G_{l}$ is a maximal compact subgroup of $G_{l}$, then $L$ is a Bruck loop of hyperbolic type (cf. [8]).

\section{Acknowledgement}

The research leading to these results has received funding from the János Bolyai Research Fellowship, from the European Union's Seventh Framework Programme (FP7/2007-2013) under grant agreements no. 318202 and no. 317721.

\section{References}

[1] A. A. Albert, Quasigroups I. Trans. Amer. Math. Soc. 54 (1943), 507519 .

[2] T. Asoh, On smooth SL(2,C) actions on 3-manifolds. Osaka J. Math. 24 (1987), 271-298.

[3] D. Betten, Die komplex-hyperbolische Ebene, Math. Z. 132, (1973), 249-259. 
[4] R. H. Bruck, Contributions to the theory of loops. Trans. Amer. Math. Soc. 60 (1946), 245-354.

[5] R. H. Bruck, A Survey of binary systems. Springer-Verlag, Berlin, Heidelberg, New York, 1971.

[6] S. S. Chen, On subgroups of the Noncompact real exceptional Lie group $F_{4}^{*}$, Math. Ann. 204 (1973), 271-284.

[7] G. Falcone, Á. Figula, K. Strambach, Multiplicative loops of quasifields with large kernel. manuscript, 2013.

[8] Á. Figula, Bol loops as section in semi-simple Lie groups of small dimension, Manuscr. Math. 121 (2006), 367-384.

[9] Á. Figula, The multiplication groups of 2-dimensional topological loops. J. Group Theory 12 (2009), 419-429.

[10] Á. Figula, On the multiplication groups of three-dimensional topological loops. J. Lie Theory 21 (2011), 385-415.

[11] R. Ghanam, I. Strugar, G. Thompson, Matrix Representation for Low Dimensional Lie Algebras. Extracta Math. 20 (2005), 151-184.

[12] K. H. Hofmann, K. Strambach, Topological and analytical loops. Quasigroups and Loops: theory and applications, 205-262, Sigma Ser. Pure Math., 8, Heldermann, Berlin, 1990.

[13] N. Knarr, Translation Planes, Springer, Berlin, Heidelberg, 1995.

[14] P. T. Nagy, K. Strambach, Loops in group theory and Lie theory. de Gruyter Expositions in Mathematics, 35. Walter de Gruyter, Berlin, 2002 .

[15] M. Niemenmaa, T. Kepka, On multiplication groups of loops. J. Algebra 135 (1990), No. 1, 112-122.

Author's address:

Institute of Mathematics, University of Debrecen,

H-4010 Debrecen, P.O.B. 12, Hungary

E-mail: figula@science.unideb.hu 